Original

\title{
Influences of mouth breathing on memory and learning ability in growing rats
}

\author{
Noriko Tsubamoto-Sano1), Junji Ohtani2), Hiroshi Ueda1), Masato Kaku1), \\ Kazuo Tanne ${ }^{1)}$, and Kotaro Tanimoto ${ }^{1)}$ \\ 1)Department of Orthodontics, Applied Life Sciences, Hiroshima University \\ Institute of Biomedical \& Health Sciences, Hiroshima, Japan \\ 2)Ohtani Dental and Orthodontics Clinic, Matsuyama, Japan
}

(Received January 12, 2018; Accepted March 23, 2018)

\begin{abstract}
This study was to investigate the influences of habitual mouth breathing on memory and learning ability during the growth period. At age 5 weeks, the experimental rats were subjected to surgery to close completely one side of the nasal cavity. An 8 -arm radial maze was used to evaluate memory and learning ability at age 7,11 , and 15 weeks. Moreover, the brain was extracted at age 7,11 , and 15 weeks, and subjected to histomorphometric examination for the distribution and number of pyramidal cells in the hippocampal CA1 and CA3 regions after Nissl staining. The trial time to accomplish each task was significantly longer in the experimental group than in the control group throughout the experimental period. The number of pyramidal cells was significantly less in the experimental rats than in controls in both the CA1 and CA3 regions for the entire experimental period. Thus, the functional deterioration of the respiratory system during the growth phase exerts a substantial effect on the growth and development of the central nervous system.
\end{abstract}

Keywords: mouth breathing; nasal respiratory obstruction; growing period.

Correspondence to Dr. Noriko Tsubamoto-Sano, Department of Orthodontics, Applied Life Sciences, Hiroshima University Institute of Biomedical \& Health Sciences, 1-2-3 Kasumi, Minami-ku, Hiroshima 734-8553, Japan

Fax:+81-82-257-5687 E-mail: norinoriko3009@gmail.com

Color figures can be viewed in the online issue at J-STAGE.

doi.org/10.2334/josnusd.18-0006

DN/JST.JSTAGE/josnusd/18-0006

\section{Introduction}

Nasal breathing is considered a physiologically normal mode of breathing in humans. The nose is located at the entrance of the respiratory system, and it performs olfaction and air conditioning in terms of the heating, humidification, and purification of inhaled air (1). Moreover, nasal respiration exerts various physiologic functions to ensure gas exchange, to maintain a stable chemical environment or blood chemistry, and to launch immunologic responses against potentially noxious agents $(2,3)$.

Structural deformities, neoplasms, and inflammatory disease in the nasopharyngeal region may increase ventilation resistance and thus complicate normal breathing. Mouth breathing is multifactorial in nature and can be induced by nasal respiratory obstructions $(4,5)$. Mouth breathing has a multifactorial etiology due to airway obstructions resulting from the hypertrophy of the pharyngeal and palatine tonsils, nasal septal deviation, nasal polyps, allergic rhinitis, and/or nasal turbinate hypertrophy (6). Subsequently, chronic and habitual mouth breathing produces reduced masticatory muscle activity and subsequent backward and downward displacements of the mandible or less-developed, distally located and hyper-divergent mandible characterized as a class II malocclusion $(7,8)$.

A previous study investigated the association between mouth breathing and nasal respiratory obstruction, which induces enhanced airway resistance and sleep-related breathing disorders (SRBD) (9-11). Moreover, SRBD is well known to induce hypoxemia, which leads to critical 


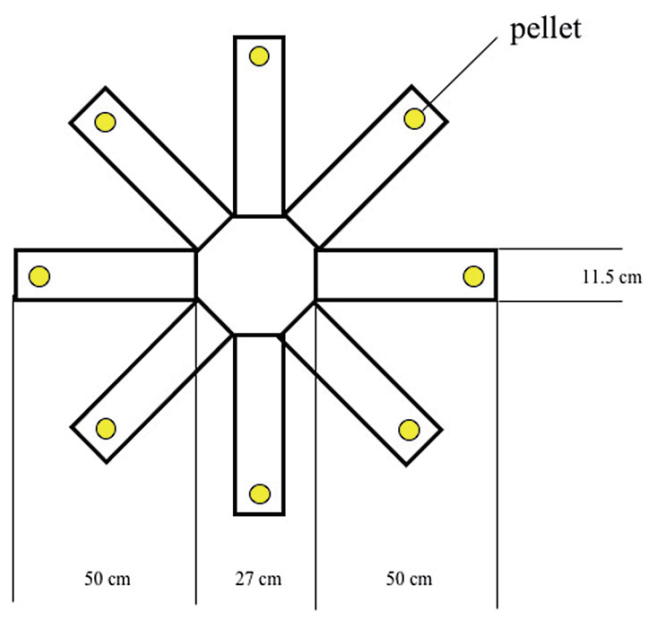

Fig. 1 Diagram of the radial arm maze task. Food pellets, which are depicted as yellow circles, are placed in a food cup at the end of each maze arm.

general health problems. If such problems persisted during the growth period, sound growth and development of the body, central nervous system, and the brain in particular might be impaired significantly.

Morris et al. reported that rats with impaired hippocampus perform poorly on the spatial cognition task (12). Moreover, previous studies demonstrated that hippocampal formation plays an especially important role in spatial memory $(13,14)$. Additionally, several morphological changes and reduction in masticatory function are induced by habitual mouth breathing during adolescent growth $(7,8,15)$; therefore, habitual mouth breathing is assumed to also affect the central nervous system responsible for memory and learning function. However, it remains unclear how mouth breathing affects the structure and function of the brain in terms of memory and learning ability.

The purpose of this study was to elucidate the influences of habitual mouth breathing on memory and learning ability in growing rats.

\section{Materials and Methods}

\section{Animals}

A total of 24 3-week-old male Wistar rats (The Jackson Laboratory, Bar Harbor, ME, USA) were used in this experiment. This study was conducted with approval from the Animal Welfare Committee of Hiroshima University (approval number: A09-83). During the experimental period, the rats were maintained at a room temperature of $22-24^{\circ} \mathrm{C}$ with a humidity of $50-60 \%$ in a 12-hour light-dark environment, and were provided with water and solid food (MF Oriental Yeast Co., Ltd., Tokyo, Japan) ad libitum. All animals were divided into experimental and control groups with 12 rats in each group. In the experimental group, the right nostril was obstructed surgically using sponges and adhesive materials (Aron alpha A, Daiichi Sankyo Co., Ltd., Tokyo, Japan) under local anesthesia with diethyl ether (Nacalai Tesque, Inc., Kyoto, Japan) at age 5 weeks. The rats in the control group underwent only local anesthesia as a sham operation.

\section{8-arm radial maze task}

Behavioral examination was assessed with an 8-arm radial maze (Neuroscience Co., Tokyo, Japan) developed by Olton and Samuelson (16) to evaluate the memory and learning ability in rats (Fig. 1). The maze consisted of a central platform $27 \mathrm{~cm}$ in diameter, with eight arms that extend radially. Each arm was $50 \mathrm{~cm}$ long and 11.5 $\mathrm{cm}$ wide and had transparent plastic side walls. Food cups were placed at the end of each arm. The maze was located in a room where many visual cues were placed close to the maze during the behavioral test. A high-speed analyzer had an automatic tracking system with a chargecoupled device camera (Watec Co., Ltd., Tsuruoka, Japan) connected to a personal computer (HewlettPackard Japan, Ltd., Tokyo, Japan), which analyzed the behavior of each rat and the time required to accomplish each task. All rats were subjected to habitual training to adapt them to the experimental environment, and were also provided access to food pellets $(50 \mathrm{mg})$ in the food cup. After 3 days of habitual training period, acquisition training was initiated in 5-week-old rats. The learning tests were performed at age 7,11, and 15 weeks. Each rat was placed gently in the center of the platform with a transparent columnar, and after $10 \mathrm{~s}$, each trial was started simultaneously. Each examination was evaluated in terms of the following parameters: 1) trial time (the learning test was ended when the rat had acquired all food pellets or when $10 \mathrm{~min}$ had elapsed without acquiring any food) and 2) achievement rate of learning test (the percentage that the learning test was finished within $10 \mathrm{~min}$ ).

\section{Histochemical examination}

The brains of the rats were removed while the rats were under deep anesthesia with pentobarbitone (Somnopentyl, Kyoritsu Seiyaku Corporation, Tokyo, Japan) when the rats were 7, 11, and 15 weeks old. The brains were rinsed with $0.1 \mathrm{M}$ phosphate-buffered saline and fixed in $4 \%$ paraformaldehyde. The specimens were then embedded in paraffin and cut into $7-\mu \mathrm{m}$-thick frontal sections. The specimens were subjected to Nissl staining. All pyramidal cells in the CA1 and CA3 regions of the left hippocampus were counted on each section according to a previously reported method (Yuzuriha T, J Keio Med 


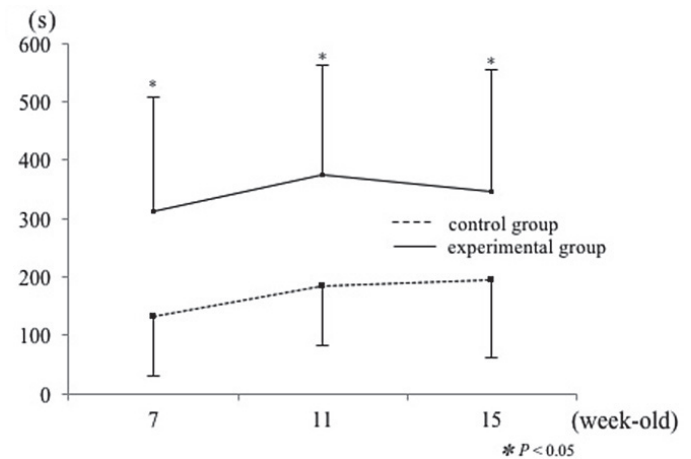

Fig. 2 Changes in the trial time evaluated by the 8 -arm radial maze test.

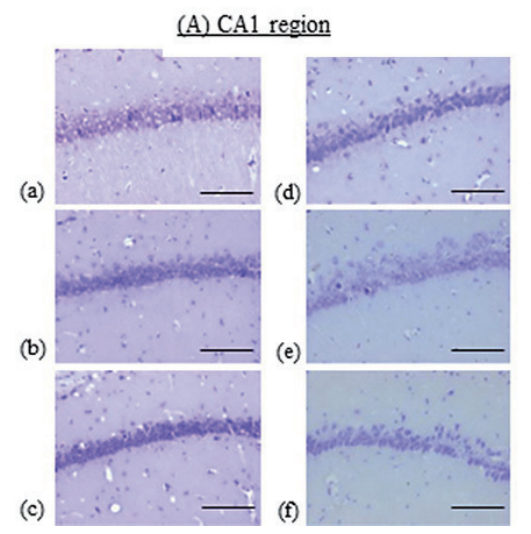

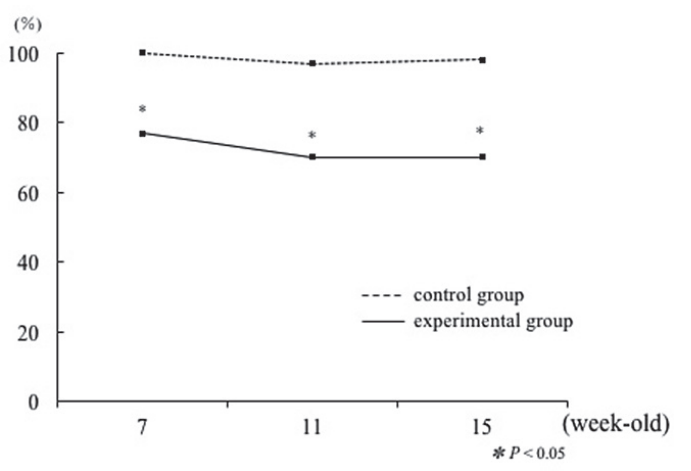

Fig. 3 Achievement rate of the learning test in the 8-arm radial maze test.

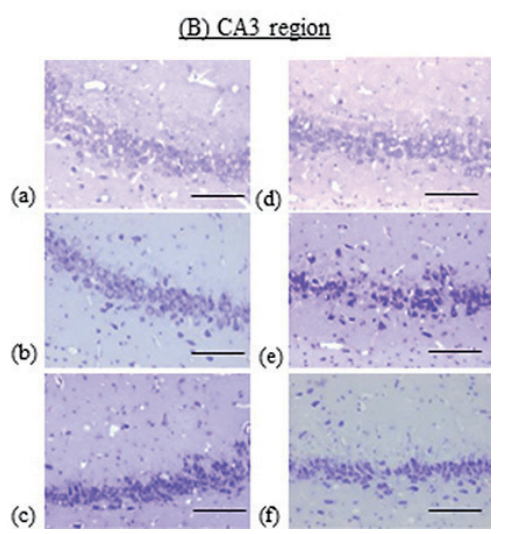

Fig. 4 (A, B) Photomicrographs showing pyramidal cells that were detected with Nissl staining in the CA1 and CA3 regions. (a) control group (7 weeks old), (b) control group (11 weeks), (c) control group (15 weeks), (d) experimental group (7 weeks), (e) experimental group (11 weeks), (f) experimental group (15 weeks) Scale bar: $100 \mu \mathrm{m}$.

Soc 67, 35-50, 1990).

\section{Blood gases analysis}

At age 7, 11, and 15 weeks, arterial blood was obtained from the left femoral artery under deep anesthesia with pentobarbitone (Somnopentyl, Kyoritsu Seiyaku Co., Tokyo, Japan). The collected blood was analyzed using a blood gas analyzer and $\mathrm{PaO} 2, \mathrm{PaCO} 2, \mathrm{HCO} 3-$, and $\mathrm{pH}$ were determined immediately.

\section{Statistical analyses}

An analysis of variances was performed to examine the differences in the number of pyramidal cells among the three age groups. A Mann-Whitney $U$-test was performed to examine the differences in the results of the behavioral tests between the experimental and control groups. Student's $t$-tests were performed to assess the mean differences in the number of pyramidal cells in the $\mathrm{CA} 1$ and $\mathrm{CA} 3$ regions between both groups and $\mathrm{PaO} 2$, $\mathrm{PaCO} 2, \mathrm{HCO} 3-$, and $\mathrm{pH}$ between both groups. All data are presented as means \pm standard deviations. $P$ values less than 0.05 were considered statistically significant.

\section{Results}

No significant differences in weight were found between the control and experimental groups during the experimental period. Thus, it is assumed that influences of a series of experimental procedures, including the obstruction of one-side nostril and local anesthesia, are not significant and serious in terms of general growth in both the experimental and control rats.

The trial time was significantly longer in the experimental group than in the control group during the experimental period (Fig. 2). The rats in the control group resolved the maze for 150-200 s compared to the experimental group, which needed twice the time, taking a significantly longer period to solve the maze. The mean success rate in the control group was very high during the experimental period: $100 \%$ at age 7 weeks, $97 \%$ at age 11 weeks, and $98 \%$ at age 15 weeks. However, the value was around $70 \%$ to $80 \%$ and significantly smaller in the experimental group, which was significantly lower than in the control group (Fig. 3).

The pyramidal cells exhibited an asymmetrical shape with a few arrays more clearly in the experimental group than in the control group in the CA1 and CA 3 regions. 


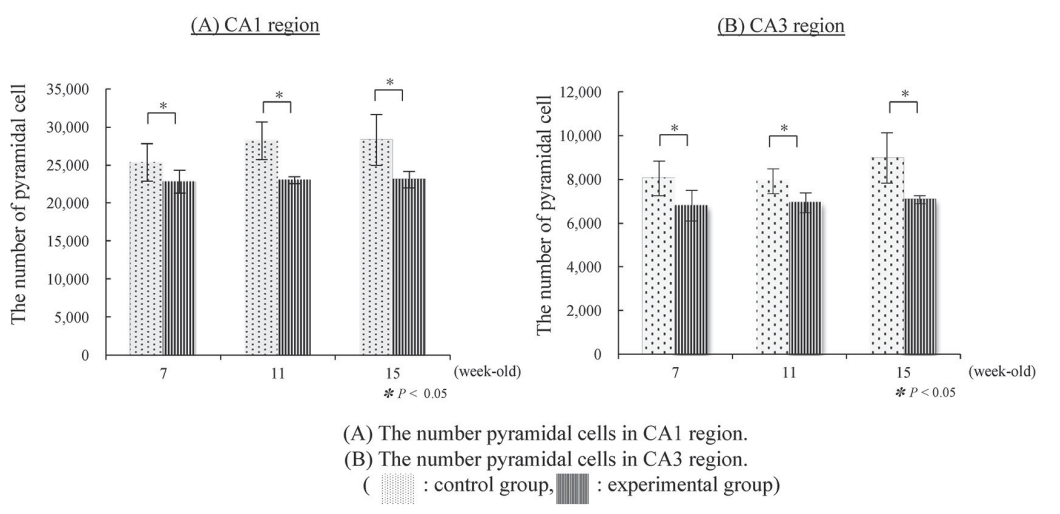

Fig. 5 (A) The number of pyramidal cells in the CA1 region. (B) The number of pyramidal cells in the $\mathrm{CA} 3$ region.

Table 1 Arterial blood gas analysis

\begin{tabular}{|c|c|c|c|c|c|}
\hline & & $\mathrm{PaO} 2(\mathrm{mmHg})$ & $\mathrm{PaCO} 2(\mathrm{mmHg})$ & $\mathrm{HCO} 3-(\mathrm{mmol} / \mathrm{L})$ & $\mathrm{pH}$ \\
\hline \multirow[t]{2}{*}{ 7-week-old } & Control group & 8.33 & 58.25 & 29.25 & 7.32 \\
\hline & Experimental group & 8.33 & 62.16 & 31.25 & 7.31 \\
\hline \multirow[t]{2}{*}{ 11-week-old } & Control group & 4.28 & 55 & 31.27 & 7.36 \\
\hline & Experimental group & 4.66 & 57 & 31.21 & 7.36 \\
\hline \multirow[t]{2}{*}{ 15-week-old } & Control group & 4.75 & \multirow{2}{*}{$\left.\begin{array}{l}59.62 \\
69.37\end{array}\right] *$} & \multirow{2}{*}{$\left.\begin{array}{l}31.11 \\
33.45\end{array}\right] *$} & 7.32 \\
\hline & Experimental group & 4.37 & & & 7.29 \\
\hline
\end{tabular}

Such tendency became more prominent at age 11 and 15 weeks (Fig. 4A, B). The number of hippocampal pyramidal cells was significantly less in the experimental group than in the control group in both the CA1 and CA3 regions (Fig. 5A, B). In the CA1 region, no substantial changes were found in the number of hippocampal pyramidal cells among the experimental groups. Although there was no significant difference, the number of pyramidal cells showed a gradual increase over time from age 7 to 15 weeks in the control group. In the CA3 region, the number of cells was invariable around 7,000 without any significant differences among the rats in experimental groups. Although there was no significant difference, the number of pyramidal cells showed an increase with age in the control group.

No significant differences were found between the control and experimental groups in all items at age 7 and 11 weeks. However, $\mathrm{PaCO} 2$ and $\mathrm{HCO} 3-$ were significantly larger in the experimental group than in the control group at age 15 weeks (Table 1). Although no significant difference was found, the $\mathrm{PaO} 2$ in the experimental group was smaller than that of the control group at age 15 weeks.

\section{Discussion}

In this study, to simulate a nasal airway obstruction and subsequent mouth breathing, only the right nostril of the rats was completely closed. Such an approach was designed to reduce surgical invasion and to allow the rats to live. Erkan et al. (17) reported that bilateral nasal obstruction in rats resulted in spontaneous death within 90-100 h after surgery. Stenosis of the nasopharyngeal airway due to epiglottis approximation is assumed to be responsible for the respiratory insufficiency in rats with nasal obstruction. In this study, all rats were alive throughout the experiment under healthy living conditions irrespective of the unilateral nasal obstruction. In addition, the body weight showed no significant differences between the experimental and control groups. These findings have thus demonstrated the validity and significance of the present mouth breathing model in growing rats.

Olton and Samuelson have described a spatial maze with eight arms radiating from a center platform wherein the animals were required to explore all arms for a pellet reward without repeating their previous choices. In their series of experiments, they revealed that rats learned this spatial discrimination task without difficultly and that their performance was not dependent on such strategies as response chaining or intra-maze scent marking (16). In addition, the 8-arm radial maze does not induce stress responses, as has been observed in Morris' water maze experiment (18). Because experiment-related stress can be reduced by acclimation for a few days prior to 
the initiation of the maze experiments (Tanaka et al., J Jpn Prosthodont Soc 8, 293-297, 1997), an acclimation period of a few days was included in this experiment to minimize the stresses induced by the maze apparatus and the surrounding environment. Such considerations have made it possible to conduct the experiments without new exercises and reduced mental anxiety and to evaluate the reaction directly related to memory and learning ability.

Concerning the brain area involved in memory, Scoville and Milner (19) have reported permanent impairment of learning and memory ability after resection of the temporal lobe including the hippocampus in patients with severe epilepsy. Since then, various studies have documented a close correlation between the hippocampus and learning ability and memory retention $(13,20)$. Cheng et al. (21) have reported a decrease in the number of pyramidal cells in the hippocampal CA1 region on days 3 and 7 after global cerebral ischemia.

In this study, a significant reduction in the total number of pyramidal cells in both the CA1 and CA3 hippocampal regions was shown in the experimental group. The following explanations could be considered. In the main excitatory hippocampal network, an information or signal is transmitted from the superficial layer (layer II) of the entorhinal cortex to the CA1 through the CA3, and the signal finally reaches the deep layers of the entorhinal cortex directly or indirectly through the subiculum (22). In this study, reduced memory and learning ability might be caused by loss or reduction in pyramidal cells in the CA3 regions and followed by reduced transmission from CA3 into the CA1 regions due to the disuse atrophy initially induced in the CA3 area.

Kurnatowski et al. have reported that children with hypertrophy of the pharyngeal tonsils attained lower marks than healthy children in neuropsychological tests (23). Fensterseifer et al. also reported that children with learning disabilities have a higher prevalence of hypertrophic tonsils or adenoids compared to children with normal school performance (24). Therefore, it has been assumed that the loss of normal nasal humidification and the warming of inspired air due to mouth breathing in these children might be responsible for changes in the diffusion capacity or viscosity of surfactant and might become a potent stimulus for bronchiolar obstruction (25). In this study, the trial time for each test was significantly longer in the experimental group than in the control group throughout the experimental period. Moreover, the achievement rate in the learning test was also significantly lower in the experimental group than in the control group throughout the experiment. From these findings, the reduced memory and learning ability assessed by the 8 -arm radial maze test may be highly related to the reduction in the number of hippocampal pyramidal cells.

Padzys et al. reported that nasal obstruction is associated with the establishment of temporary oral breathing, which causes delay of lung development in rats (26). Furthermore, $\mathrm{PaO} 2$ values decrease and $\mathrm{PaCo} 2$ values increase when mouth breathing is caused by bilateral nasal obstruction in humans and animals (27-32). These results are considered to be caused by a decrease in lung capacity and an increase in residual capacity as a result of nasal respiratory obstruction.

In this study, because of the unilateral nasal obstruction, there was no significant difference in $\mathrm{PaO} 2$ throughout the experimental period. However, $\mathrm{PaCO} 2$ and $\mathrm{HCO} 3$ - increased significantly in the experimental group at age 15 weeks. Lung compliance is decreased by long-term mouth breathing, which in turn is caused by the ventilatory impairment. A decrease in the blood oxygenation levels induced by habitual mouth breathing may also be relevant to these functional and neuropathological changes; however, the mechanisms underlying the reduction in the hippocampal pyramidal cells remain unknown and require further investigation with cellular and molecular examination.

In addition, early temporary nasal obstruction associated with forced oral breathing causes long-term craniofacial growth retardation (26). Hsu and Yamaguchi have reported that mouth breathing decreases the number of chewing cycles and chewing activity in terms of the degree and duration of EMG activity of the masseter muscle (33). Tsutsui et al. demonstrated that a long-term reduction or defect in masticatory afferent stimulation induces impairments in memory and learning function (34). From these results, a decrease in occlusal and masticatory stimuli due to mouth breathing might have a relationship with reduced learning ability and memory retention.

In conclusion, mouth breathing during the growing period results in a considerable reduction in memory and learning ability in rodents. Thus, functional deterioration of the respiratory system during the growth phase is considered to exert a substantial effect on the growth and development of the central nervous system.

\section{Acknowledgments}

The authors thank Cynthia Concepcion for valuable advice. This study was supported by a Grant-in-Aid for Scientific Research from the Japan Society for the Promotion of Science (23792430).

\section{Conflict of interest}

The authors have no conflict of interest to declare. 


\section{References}

1. Geurkink N (1983) Nasal anatomy, physiology, and function. J Allergy Clin Immunol 72, 123-128.

2. Grodins FS, Buell J, Bart AJ (1967) Mathematical analysis and digital simulation of the respiratory control system. J Appl Physiol 22, 260-276.

3. Saunders KB, Bali HN, Carson ER (1980) A breathing model of the respiratory system; the controlled system. J Theor Biol 84, 135-161.

4. Ricketts RM (1968) Respiratory obstruction syndrome. Am J Orthod 54, 495-507.

5. Watson RM Jr, Warren DW, Fischer ND (1968) Nasal resistance, skeletal classification, and mouth breathing in orthodontic patients. Am J Orthod 54, 367-379.

6. Emslie RD, Massler M, Zwemer JD (1952) Mouth breathing. I. Etiology and effects; a review. J Am Dent Assoc 44, 506-521.

7. McNamara JA (1981) Influence of respiratory pattern on craniofacial growth. Angle Orthod 51, 269-300.

8. Yamada T, Tanne K, Miyamoto K, Yamauchi K (1997) Influences of nasal respiratory obstruction on craniofacial growth in young Macaca fuscata monkeys. Am J Orthod Dentofacial Orthop 111, 38-43.

9. McNicholas WT, Tarlo S, Cole P, Zamel N, Rutherford R, Griffin D et al. (1982) Obstructive apneas during sleep in patients with seasonal allergic rhinitis. Am Rev Respir Dis 126, 625-628.

10. Lavie P, Fischel N, Zomer J, Eliaschar I (1983) The effects of partial and complete mechanical occlusion of the nasal passages on sleep structure and breathing in sleep. Acta Otolaryngol 95, 161-166

11. Virkkula P, Maasilta P, Hytönen M, Salmi T, Malmberg H (2003) Nasal obstruction and sleep-disordered breathing: the effect of supine body position on nasal measurements in snorers. Acta Otolaryngol 123, 648-654.

12. Morris RG, Garrud P, Rawlins JN, O'Keefe J (1982) Place navigation impaired in rats with hippocampal lesions. Nature 297, 681-683.

13. Weiskrantz L, Warrington EK (1975) Some comments on Wood's and Piercy's claim of a similarity between amnesic memory and normal forgetting. Neuropsychologia 13, 365-368.

14. Jarrard LE (1978) Selective hippocampal lesions: differential effects on performance by rats of a spatial task with preoperative versus postoperative training. J Comp Physiol Psychol 92, 1119-1127.

15. Onozuka M, Watanabe K, Mirbod SM (1999) Reduced mastication stimulates impairment of spatial memory and degeneration of hippocampal neurons in aged SAMP8 mice. Brain Res 826, 148-153.

16. Olton DS, Samuelson RJ (1976) Remembrance of places passed: spatial memory in rats. J Exp Psychol Anim Behave Process 2, 97-116.

17. Erkan M, Erhan E, Sağlam A, Arslan S (1994) Compensatory mechanisms in rats with nasal obstructions. Tokai J Exp Clin
Med 19, 67-71.

18. Morris R (1984) Developments of a water-maze procedure for studying spatial learning in the rat. J Neurosci Methods $11,47-60$

19. Scoville WB, Milner B (1957) Loss of recent memory after bilateral hippocampal lesions. J Neurol Neurosurs Psychiatry 20, 11-21.

20. Mishkin M (1978) Memory in monkeys severely impaired by combined but not by separate removal of amygdala and hippocampus. Nature 273, 297-298.

21. Cheng O, Ostrowski RP, Wu B, Liu W, Chen C, Zhang JH (2011) Cyclooxygenase-2 mediates hyperbaric oxygen preconditioning in the rat model of transient global cerebral ischemia. Stroke 42, 484-490.

22. Andersen P, Bliss TV, Lomo T, Olsen LI, Skrede KK (1969) Lamellar organization of hippocampal excitatory pathways. Acta Physiol Scand 76, 4A-5A.

23. Kurnatowski P, Putynski L, Lapienis M, Kowalska B (2006) Neurocognitive abilities in children with adenotonsillar hypertrophy. Int J Pediatr Otorhinolaryngol 70, 419-424.

24. Fensterseifer GS, Carpes O, Weckx LL, Martha VF (2013) Mouth breathing in children with learning disorders. Braz J Otorhinolaryngol 79, 620-624.

25. G.E. Woodson, K.T. Robbins (1985) Nasal obstruction and pulmonary function: The role of humidification. Otolaryngol Head Neck Surg 93, 505-511.

26. Padzys GS, Tankosic C, Trabalon M, Martrette JM (2012) Craniofacial development and physiological state after early oral breathing in rats. Eur J Oral Sci 120, 21-28.

27. Cook TA, Komorn RM (1973) Statistical analysis of the alterations of blood gases produced by nasal packing. Laryngoscope 83, 1802-1809.

28. Ogura JH, Nelson JR, Suemitsu M, Kawamoto S (1973) Relationship between pulmonary resistance and changes in arterial blood gas tension in dogs with nasal obstruction and partial laryngeal obstruction. Ann Otol Rhinol Laryngol 82, 668-683.

29. Cavo JW, Kawamoto S, Berlin BP, Zollinger W, Ogura JH (1975) Arterial blood gas changes following nasal packing in dogs. Laryngoscope 85, 2055-2068.

30. Krajina Z, Cepelja I, Suvajdzić S (1977) Nasal obstruction and cardiopulmonary system in children. Acta Otolaryngol $83,40-46$

31. Cvetnic V, Cvetnic S, Grabac I (1981) Temporary artificial obstruction of the nose and changes in gas exchange in the blood. Laryngoscope 91, 1001-1009.

32. Ramadan MF (1983) Experimental nasal obstruction and changes in the arterial blood gases. Clin Otolaryngol 8, 245-250.

33. Hsu HY, Yamaguchi K (2012) Decreased chewing activity during mouth breathing. J Oral Rehabil 39, 559-567.

34. Tsutsui K, Kaku M, Motokawa M, Tohma Y, Kawata, T, Fujita $\mathrm{T}$ et al. (2007) Influences of reduced masticatory sensory input from soft-diet feeding upon spatial memory/ learning ability in mice. Biomed Res 28, 1-7. 